\title{
ФУНКЦИОНАЛЬНОЕ МОДЕЛИРОВАНИЕ ПРОЦЕССА МОНИТОРИНГА МНЕНИЯ ОБУЧАЮЩИХСЯ
}

\section{FUNCTIONAL MODELING \\ OF THE PROCESS OF MONITORING THE OPINION OF STUDENTS}

\section{T. Gerashenkova Yu. Sinyukova}

Summary. The work is devoted to the development of a functional model of the monitoring process of students' opinions. The urgency of monitoring the students' opinion has been substantiated, the main blocks of the monitoring process have been designed, and a BPMN diagram has been built.

Keywords: monitoring, management, students' opinion, management decision making.

\author{
Геращенкова Татьяна Михайловна \\ Д.э.н., профессор, ФГБОУВО «Брянский \\ государственный технический университет» \\ gerash-tatyana@yandex.ru \\ Синюкова Юлия Александровна \\ Аспирант, ФГБОУ ВО «Брянский государственный \\ технический университет» \\ ulya16-96@mail.ru
}

Аннотация. Работа посвящена разработке функциональной модели процесса мониторинга мнения обучающихся. Обоснована актуальность проведения мониторинга мнения обучающихся, спроектированы основные блоки процесса мониторинга, построена BPMN-диаграмма.

Ключевые слова: мониторинг, управление, мнение обучающихся, принятие управленческих решений.

вать на изменения запросов обучающихся, учитывать их мнение при принятии управленческих решений. Роль мнения обучающихся в повышении эффективности деятельности образовательного учреждения исследована в [4].

Для того чтобы принимать управленческие решения с учетом мнения обучающихся, лицо, принимающее решение, необходимо обеспечить актуальной информацией о потребностях обучающихся, их ожиданиях, предложениях, уровне удовлетворенности качеством образовательных услуг и условиями их предоставления, т.е. получать постоянную обратную связь от обучающихся. Многие зарубежные и отечественные образовательные организации понимают необходимость учета мнения обучающихся и активно вовлечены в этот процесс [3], однако эпизодичность и нерегулярность проведения учета мнения обучающихся и отсутствие четких механизмов применения его результатов не позволяют развивать методологию и методики проведения исследования мнения обучающихся с учетом стратегических задач образовательного учреждения [5, с. 117].

Инструментом, который позволяет постоянно наблюдать и эффективно проводить анализ управляемой системы, в данном случае - обучающихся, является мониторинг.

Таким образом, целью представленной работы является разработка функциональной модели мониторинга мнения обучающихся. 


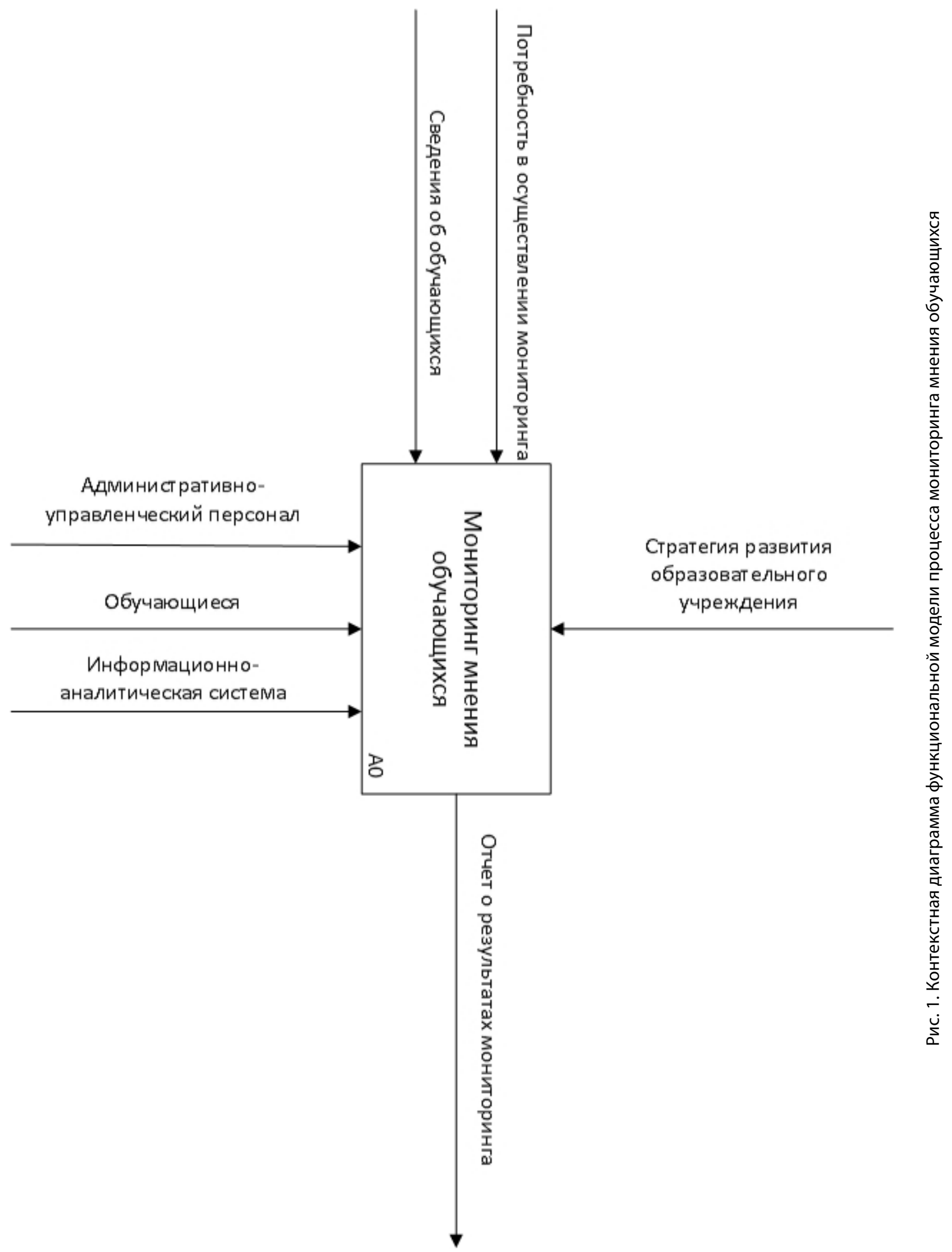




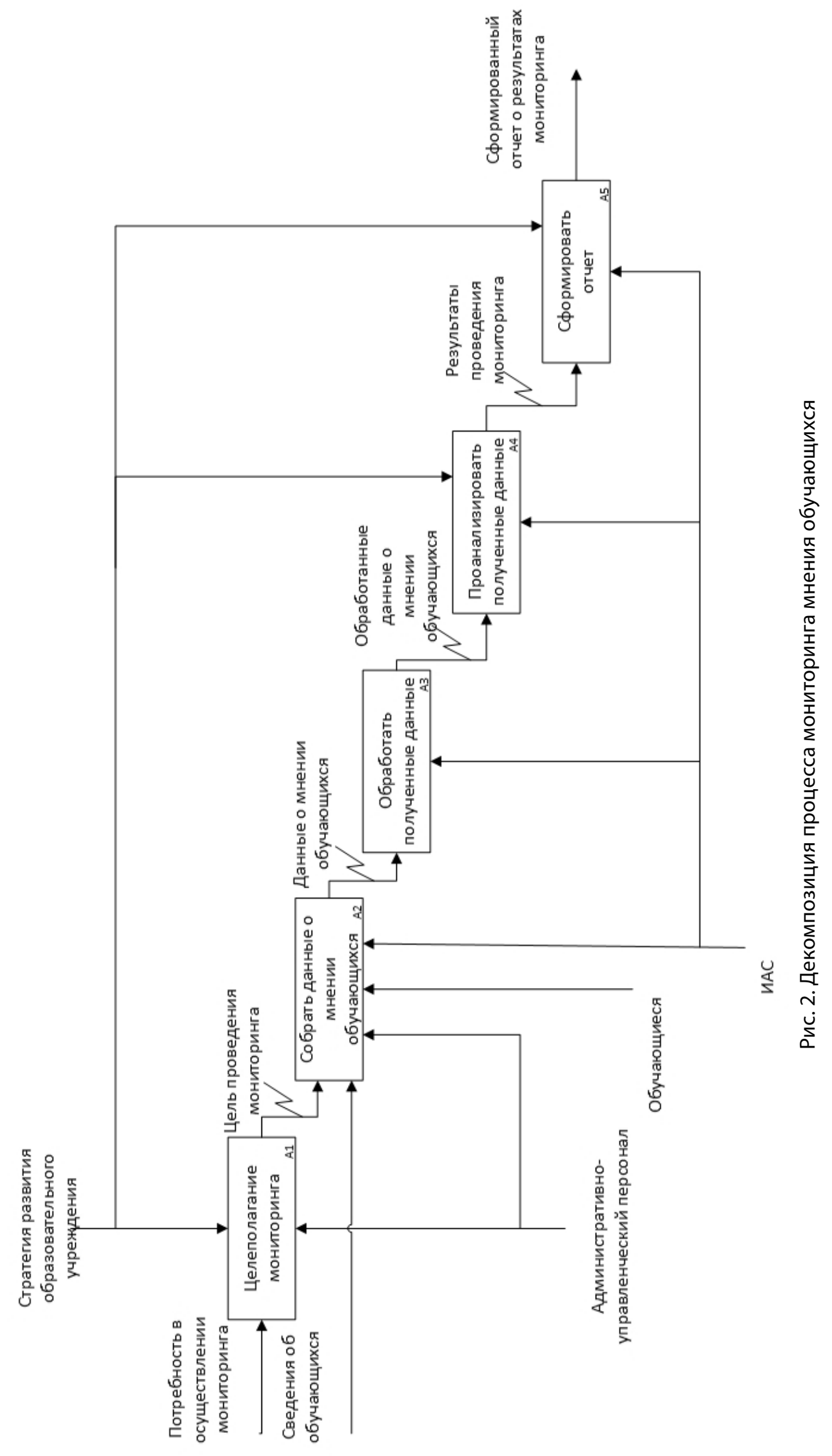




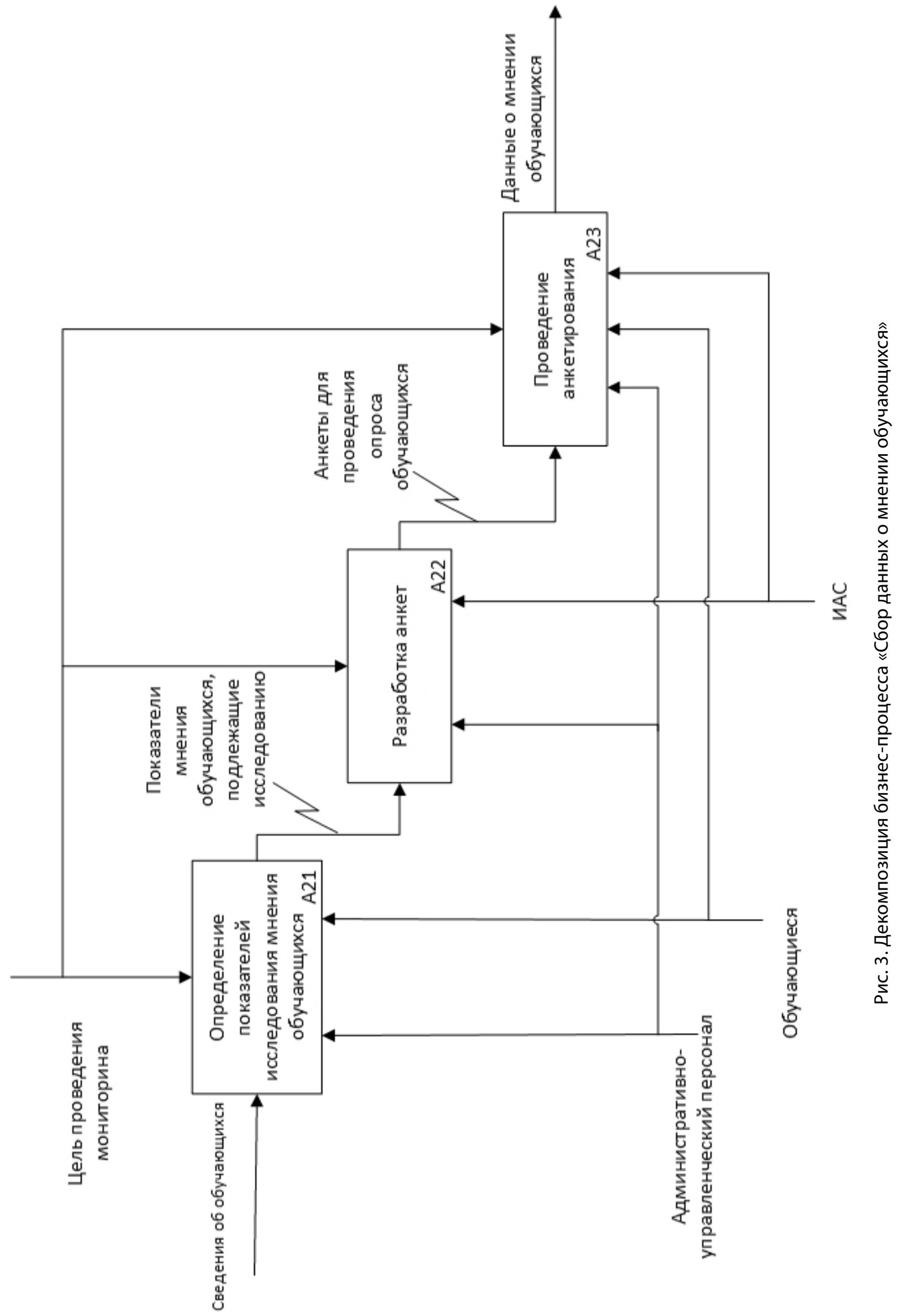




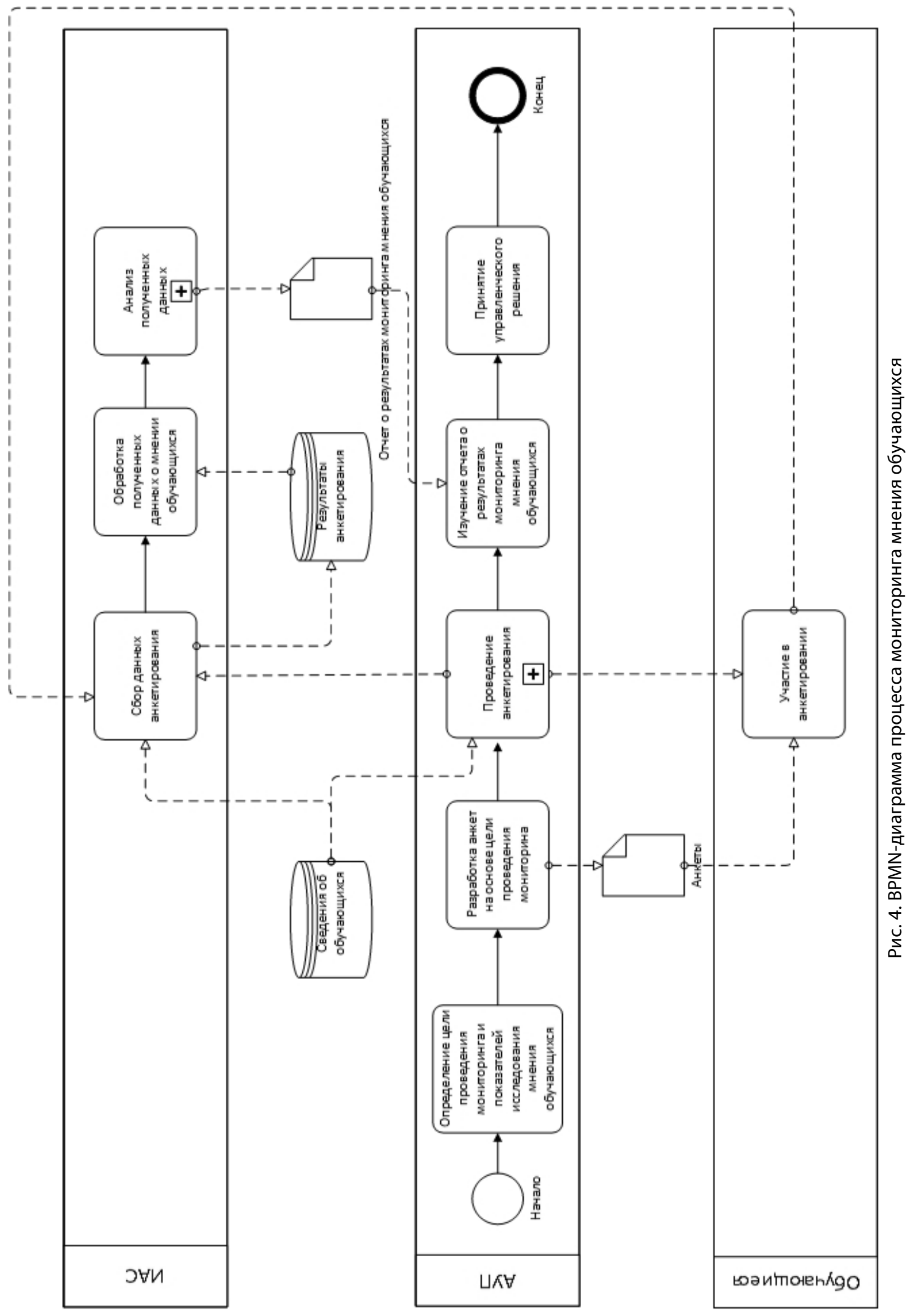




\section{Молемирование прошесса}

мониторинга мнения обучаюшихся

На основе изучения обобщенной функциональной модели мониторинга данных в социально-экономических системах [6] были спроектированы основные блоки процесса мониторинга мнения обучающихся.

На рисунке 1 представлены основные элементы модели процесса мониторинга мнения обучающихся. Фактором управления в данном случае является стратегия развития образовательного учреждения. В качестве механизмов выступают административно-управленческий персонал (АУП) образовательного учреждения, сами обучающиеся и информационно-аналитическая система (ИАС). Исходной информацией являются конкретная потребность в проведении мониторинга и сведения об обучающихся. Результатом работы системы мониторинга мнения обучающихся является отчет о результатах исследования, содержащий необходимую для принятия управленческого решения по поставленной цели проведения мониторинга информацию.

Рис. 2 отображает декомпозицию процесса мониторинга мнения обучающихся в пять этапов. В начале необходимо определить цель проведения мониторинга в соответствии со стратегией развития образовательного учреждения (например, изучить мнение обучающихся относительно организации их научно-исследовательской деятельности на текущей момент), затем на основе сведений об обучающихся с использованием ИАС провести сбор, обработку и анализ данных о мнении обучающихся и сформировать отчет.

На рисунке 3 представлена декомпозиция бизнес-процесса «Сбор данных о мнении обучающихся», отражающая последовательность работ при его реализации.

После формирования отчета о результатах проведения мониторинга мнения обучающихся по заложенным в цели проведения мониторинга показателям с рекомендациями и предложениями по их совершенствованию, данные передаются АУП, который с учетом этих сведений принимает управленческие решения. После реализации управленческих решений с целью проверки их эффективности необходимо повторно провести мониторинг мнения обучающихся по тем же показателям.

Рис. 4 иллюстрирует последовательность рабочих действий, распределение зон ответственности и перемещение информационных потоков, необходимых для выполнения процесса мониторинга мнения обучающихся.

\section{Зак^ючение}

Анализ литературы показал, что многие образовательные организации осознают необходимость и актуальность проведения мониторинга мнения обучающихся как источника информации для принятия управленческих решений и оценки их эффективности. Однако в настоящее время отсутствуют четкие механизмы проведения исследования мнения обучающихся с учетом стратегии развития образовательного учреждения и применения результатов данного исследования.

В работе спроектированы основные блоки функциональной модели процесса мониторинга мнения обучающихся, а также построена BPMN-диаграмма процесса.

\section{ЛИТЕРАТУРА}

1. Бахтин М.Б., Тормосова А.К. Эффективность деятельности образовательных организаций и их руководителей: региональная и муниципальная практика // Человек и образование.— 2020.— № 4 (65). — С. 139-146.

2. Пирайнен Е.В. Трансформация образовательного процесса в современном информационном пространстве // Дискурс.— 2017. — № 4. - С. 32-36.

3. Кормина Е.Я., Наумова А.А. Современные тенденции мониторинга удовлетворенности обучающихся качеством образования. Практика зарубежных и отечественных организаций // Вестник Сургутского государственного педагогического университета. — 2015. — № 2 (35). — C. 110-126.

4. Синюкова Ю.А. Исследование роли мнения обучающихся в повышении эффективности деятельности образовательного учреждения // Новые горизонты: материалы VII научно-практической конференции с международным участием, 20 марта 2020 года, Брянск/под ред. 0.М. Голембиовской. Брянск: БГТУ, 2020.- - С. 746-749.

5. Сущенко А.Д., Сандлер Д.Г. Как студенты вовлечены в механизмы «обратной связи»: системная практика исследований в УРФу // Университетское управление: практика и анализ. — 2017. — № 2 (108). — С. 176-191.

6. Копелиович Д.И., Рыженков Д.А. Функциональное моделирование процесса мониторинга данных // Мониторинг. Наука и технологии. — 2016. — № 1 (26). - C. 49-53. 